\title{
Standardization of Airport Architectural Design Projects BIM-based for Code Checking
}

XXIV International Conference of the Iberoamerican Society of Digital Graphics Medellin | Colombia

\author{
Ítalo Guedes dos Santos \\ Universidade Federal de Pernambuco | Brasil | italo.guedes@ufpe.br \\ Max Lira Veras Xavier de Andrade \\ Universidade Federal de Pernambuco | Brasil | max.andrade@ufpe.br
}

\begin{abstract}
This paper addresses the use of BIM for code verification and automatic validation of the Architectural Design of Airports (ADA). In Brazil, the evaluation and approval of ADA are carried out by INFRAERO. Currently, designs are evaluated manually, resulting in errors and long evaluation time. To deal with this problem, a conceptual framework for automated ADA assessment with Code Checking is proposed. The method used was Design Science Research, with the proposal of an artifact. The partial results show the importance of establishing protocols for BIM modeling, based on IFC as an important tool for automated assessment with code checking.
\end{abstract}

Keywords: Airports; Architectural Design of Airport; Building Information Modeling; Code Checking; IFC.

\begin{abstract}
INTRODUÇÃO
Uma das maneiras de demonstrar o quão complexo são os Projetos Arquitetônicos Aeroportuários (PAA) é pela grande quantidade de requisitos técnicos que esses precisam atender para serem aprovados pelos órgãos competentes. No Brasil, a avaliação e a aprovação dos PAAs são realizadas pela INFRAERO (empresa pública federal brasileira responsável pela administração de diversos aeroportos no país), SAC (Secretaria de Aviação Civil), dentre outros órgãos competentes. No cenário atual, a equipe de projetistas desenvolve o PAA e o submete a um processo de validação manual realizado pela equipe de analistas. Tais processos tendem a ser demorados (perdurando meses ou até anos). Além disso, os processos de avaliação são subjetivos nas análises, devido às diferentes perspectivas e expertises de conhecimento, específicos de cada analista no momento da análise. Em resposta a esse problema, este artigo analisa como o BIM pode aperfeiçoar esses processos, minimizando erros de avaliação do PAA, reduzindo o tempo de avaliação do projeto e evitando possíveis retrabalhos, tanto por parte dos analistas quanto por parte dos projetistas de PAA.
\end{abstract}

O uso do BIM vinculado a esse tipo de atividade é denominado de Code Checking (CC). De acordo com Estman et al. (2009), CC é definido como um processo de avaliação de relacionamentos ou atributos de projeto com base em suas configurações de objetos. O uso do Modelo BIM para o CC pode possibilitar um novo cenário para os analistas de PAA automatizarem o processo de avaliação, reduzindo o tempo de análise e aumentando a eficiência em relação ao processo de validação tradicional (Benning et al., 2010).

Um exemplo de regras testadas e demonstradas por Guedes e Andrade (2019) é a regra que estabelece a largura mínima de circulação entre filas de check-in e balcão de atendimento no PAA. Nesse caso, não só foi necessário criar uma regra em um software de avaliação BIM, mas também foi necessário que o Modelo BIM de autoria (modelo do projeto arquitetônico) que carrega essas informações tivesse sido criado em uma Plataforma BIM e exportado via IFC com as informações corretas, tais como, Nome do Espaço (IfcSpace), Largura do Bouding Box Width (IfcSpace), assim como os parâmetros específicos para que o software de análise pudesse validar a regra de maneira correta. O que é importante observar é que no processo de validação da qualidade de um projeto é fundamental considerar não só a qualidade da regra criada, mas também a qualidade do modelo criado, que deve pendurar as informações do modelo seguindo certas prescrições, necessárias para que essas sejam reconhecidas pelo software de avaliação BIM. Nesse sentido, este artigo discute e propõe um modelo conceitual (ainda em desenvolvimento) de uso de CC para o processo de avaliação de PAA a ser realizado pelos órgãos competentes. Discute também sobre a criação de regras de validação, diretrizes para modelagem e exportação de modelos de PAA para a avaliação. Por fim, analisa os tipos de regras mais usadas, e, mostra a importância em classificar e selecionar essas classes de regras de modo a tornar mais eficiente $\mathrm{o}$ processo de modelagem e aprovação de PAA.

\section{METODOLOGIA}

O método utilizado na pesquisa é o Design Science Research (March e Smith, 1995). Este, segundo March e Smith (1995), constitui-se em duas macro etapas: (1) Construção, etapa destinada ao desenvolvimento do artefato; e, (2) Avaliação do Artefato, etapa destinada a mensuração da eficiência do artefato, a partir da verificação do seu comportamento no contexto analisado. Este artigo abordará a etapa (1). 
A etapa (1) de Construção do Artefato constitui-se em duas fases. A primeira fase refere-se à fundamentação teórica sobre o tema. Iniciou-se essa fase com uma Revisão Sistemática de Literatura (RSL). Foram identificadas publicações internacionais e nacionais a respeito da temática nos últimos 10 anos. Ainda na primeira fase estão sendo realizados estudos de casos em processos de análise e validação de projetos aeroportuários no Brasil, junto à INFRAERO, SAC e empresas especializadas no desenvolvimento de PAA. A segunda fase da etapa (1) consiste na concepção e desenvolvimento do artefato. Essa fase iniciou com o mapeamento das diretrizes de avaliação de PAA estabelecidas pelos órgãos competentes. Em seguida, essas diretrizes estão sendo transcritas em regras que estão sendo implementadas em softwares de avaliação BIM e testadas em situações de projeto. Entre os vários experimentos realizados estão a avaliação de boas práticas de modelagem de PAA e a avaliação da eficiência em implementar cada uma dessas regras, a partir da classificação das regras.

O objetivo da segunda fase da etapa (1) consistiu em transpor as principais normas brasileiras em critérios que possam ser inseridos em softwares de avaliação automática baseados no BIM com a função code checking e validation (Succar, 2015). A partir de alguns teste em softwares e de experiências da literatura, que aponta para o uso mais sistemático do Solibri Model Checker (SMC) em locais como Singapura (Corenet), Noruega (Stasbygg), Australia (CRC), EUA (ICC e GSA) (Eastman et al. 2009), esta pesquisa optou por realizar as experimentações utilizando esse software. Uma vez definido o software, estão sendo criadas e testadas as regras de checagem no $\mathrm{SMC}$ em um projeto brasileiro de PAA modelado em um software de autoria BIM. maneiras mais eficientes de construção do modelo BIM desenvolvido pela equipe de projetistas de PAA, sem reduzir a capacidade de projeto e de modo a pendurar as informações necessárias e suficientes para a avaliação do projeto, com precisão. Ou seja, quais as informações que precisam estar incorporadas nos modelos BIM (características de modelagem) para que possam ser avaliadas corretamente no SMC. Também estão sendo consideradas as terminologias usadas nas regras e sua classificação. A investigação feita nesse trabalho tem como base o uso de um esquema de comunicação da informação aberto publicamente que é o Industry Foundation Classes (IFC). O IFC tem sido aceito como único padrão aberto e relativamente maduro suportado atualmente pelos principais aplicativos BIM.

As etapas usadas no desenvolvimento do artefato são: identificação das principais diretrizes usadas na avaliação de PAA pelos órgão competentes; transcrição das diretrizes em regras; definição de diretrizes para a criação do Modelo BIM a ser desenvolvido pela equipe de projetistas de PAA, de modo a ser possível a avaliação automática em software BIM (aqui inclui a definição das propriedades IFC que devem estar penduradas no modelo, as terminologias, os filtros para exportação com os MVDs usados); definição dos procedimentos para avaliação da consistência do modelo (com base nas diretrizes de modelagem); e, verificação da eficiência da avaliação. Entre as etapas que estão sendo estudadas também inclui a análise e classificação de cada uma das regras, de acordo com as classes de regras definidas por Solihin e Eastman (2015). Essa classificação será importante para identificar quais classes de regras trazem mais benefícios em termos de facilidade e eficiência para implementação nos processos de avaliação automática baseada no CC.

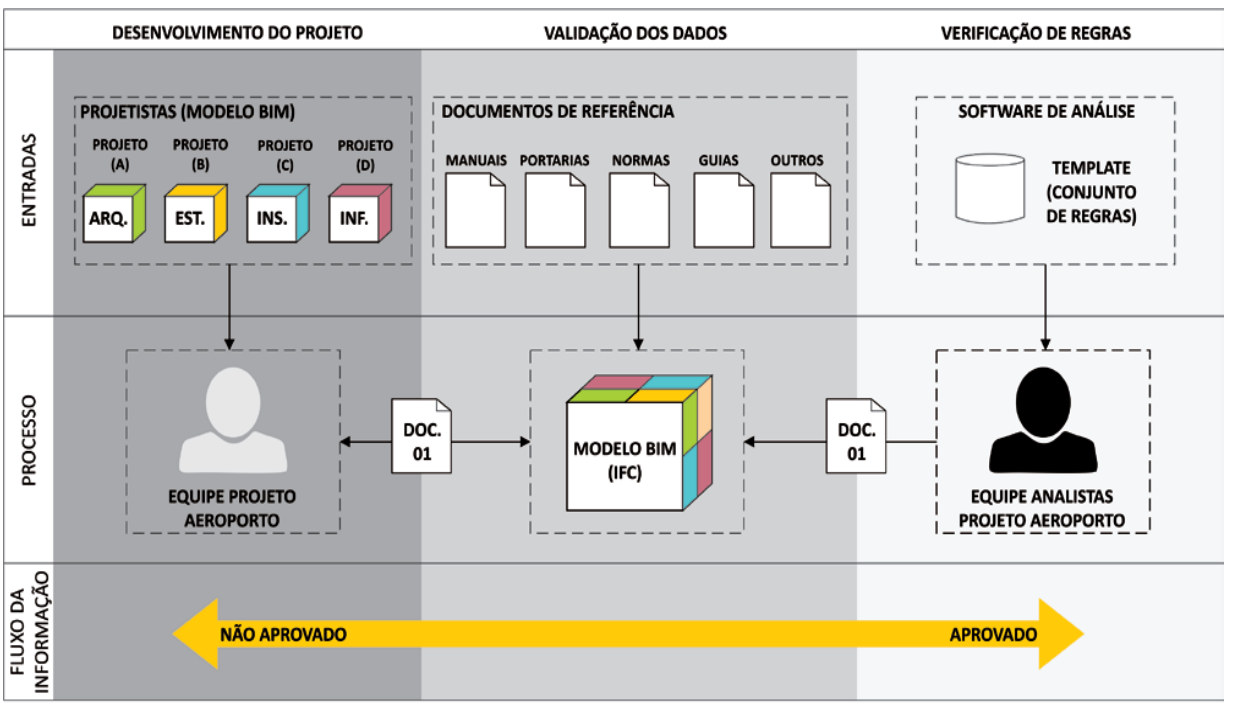

\section{LEGENDA (DOCUMENTOS):}

DOC.01: MANUAL DE DIRETRIZES (PADRONIZAÇÃO DO MODELO BIM)
LEGENDA (SÍMBOLOS):

Xx. - MODELO BIM D - DOCUMENTOS

- TEMPLATE (SOFTWARE DE ANÁLISE)

Figura 1: Síntese das etapas do processo de projeto e avaliação de PAA abordados na pesquisa. Fonte: Elaborado pelos autores (2020).

O que está sendo analisado não é apenas quais tipos de regras que podem ser implementadas e como implementalas em softwares de avaliação BIM, mas, também,
A Figura 1 apresenta uma síntese das etapas do processo de projeto e avaliação de PAA que estão sendo tratadas pelo artefato desta pesquisa. A primeira fase 
(Desenvolvimento do Projeto) consiste no desenvolvimento do projeto de PAA em um software de autoria BIM (Plataforma BIM). Nessa etapa a equipe de projetistas desenvolvem um Modelo de Informação da Construção em uma Plataforma BIM (Revit, Archicad, OpenBuilding ou outra) com base no Manual de Diretrizes para a Modelagem (MDM), que deverá ser estabelecido pelos órgãos competentes responsáveis pela avaliação de PAA no Brasil (estágio em desenvolvimento). A segunda fase (Validação dos Dados) será realizada pela equipe de analistas, consiste na avaliação da qualidade do modelo IFC fornecido pelas equipes de projetistas de PAA. A última fase (Verificação de Regras) expressa-se na etapa de avaliação da qualidade da solução, em termos de atendimento as condicionantes levadas em consideração para aprovação de um PAA junto aos órgãos competentes por meio da verificação de regras. Essas fases propostas no artefato, que vem sendo desenvolvidas por essa pesquisa, indicam uma mudança no processo de avaliação do projeto, que passa a ser automatizado, a partir de diretrizes estabelecidas em projeto. O que se espera com o artefato é uma redução do tempo de avaliação do projeto por parte da equipe de analistas, tendo em vista a automatização na fase de avaliação, assim como, uma maior padronização no processo de avaliação de PAA no Brasil, uma vez que o modelo BIM exportado via IFC, aliado ao software de análise, passam a auxiliar o analista no processo de avaliação do PAA.

Este artigo irá investigar mais especificamente a relevância do Manual de Diretrizes para a Modelagem (MDM), que é um instrumento importante para subsidiar o desenvolvimento do modelo BIM a ser concebido pela equipe de projetista de PAA. A partir de uma modelagem baseada nas diretrizes estabelecidas nesse manual, será possível validar os dados do modelo IFC que serão usados para avaliação pelas equipes de analistas de PAA. Esses dados constituem-se nas informações pertinentes e necessárias para a verificação de regras automáticas (Code Checking) na fase de avaliação da solução (Verificação das Regras).

\section{PROJETOS AEROPORTUÁRIOS}

Em tempos atrás o projeto de um aeroporto era traçado especificamente conforme exigências das aeronaves que iriam operar naquele aeroporto. Este demandava de um limitado programa de necessidades. Atualmente, em consequência de diversos fatores, tais como a proximidade com centros geradores de demanda, multifuncionalidade de serviços, surgimento de novos conceitos, tais como: aeroshopping, aeroporto cidade, aeroporto industrial, assim como o surgimento de novos materiais e processos construtivos, fizeram com que o desenvolvimento de um PAA se transformasse em uma atividade complexa. Isso faz exigir conhecimento especializado para a concepção e desenvolvimento do projeto. O alto nível de complexidade desse tipo de empreendimento pode ser visto na estrutura funcional do edifício, que é dotado de vida própria, na complexidade nas relações com o seu entorno imediato, bem como, na adequação à diversos parâmetros, como, níveis de serviço, níveis de segurança, adequação ao avanço de novas tecnologias. É importante esclarecer que esse artigo abordará projetos aeroportuários e não aeródromos. Segundo o Art.27 do Código Brasileiro de Aeronáutica (1986), "aeródromo é toda área destinada a pouso, decolagem e movimentação de aeronaves", podendo ser classificado como civis e militares. A diferença entre aeródromos e aeroportos, é que os aeroportos apresentam instalações e facilidades específicas de infraestrutura básica para operações de aeronaves, embarque e desembarque de pessoas e cargas. Em resumo, a diferença entre aeródromo e aeroporto é a existência ou não da infraestrutura básica de instalações e facilidades.

No que se refere ao desenvolvimento de um projeto de aeroportuário no Brasil, existe uma série de pré-requisitos normativos que norteiam o projetista no momento do desenvolvimento de um projeto aeroportuário. Dentre os documentos, pode-se destacar: a) RBAC (Regulamentos Brasileiros da Aviação Civil), b) Portaria 1141/GM5, c) Anexo 14, d) Manual de Critérios e Condicionantes de Planejamento Aeroportuários (INFRAERO), dentre outros de documentos de referências. Faz-se necessário que a equipe de projetistas tenha expertise para conceber um projeto em atendimento a todas as normas vigentes.

Com a incorporação do Code Checking no processo de projeto aeroportuário é possível que a equipe de analistas dos projetos no Brasil possa tirar proveito de uma verificação automatizada (Code Checking), através do uso de um software que avalia um conjunto de regras sobre o modelo de informação do projeto do aeroporto. Mas, para isso, se faz necessário que esse modelo contenha as informações necessárias e suficientes, e que utilize um protocolo de troca de informações capaz de levar a informação certa e no local certo, utilizando um esquema que seja aceito internacionalmente. Este trabalho baseará os estudos no uso do esquema IFC como protocolo de troca de informações entre a etapa de síntese (desenvolvimento do projeto de arquitetura) e avaliação do artefato arquitetônico (realizada pela equipe de analistas). Busca-se, então, padronizar os procedimentos na criação das entidades IFC a serem verificadas no processo de avaliação de projeto aeroportuário de arquitetura, de modo que seja confiável o uso da verificação automática para identificar se o projeto atende ou não aos requisitos preestabelecidos em normas.

\section{PROCESSO DE PROJETOS AEROPORTUÁRIOS EM BIM}

O processo de projeto aeroportuário baseado no BIM é definido pela integração multidisciplinar de dados do projeto, sua organização e os processos envolvidos para o seu desenvolvimento. O projeto deve ser concebido agregando-se todas as informações relevantes e necessárias em cada fase do PAA. $O$ produto final deve ser um banco de dados pendurado em um modelo tridimensional, com as informações necessárias e suficientes para o desenvolvimento do projeto do edifício. Dependendo do uso BIM, o modelo digital poderá conter as propriedades definidoras de seus componentes, seus materiais, características específicas, códigos de serviços associados as suas execuções, ciclo de manutenção, parâmetros de quantidades, custos, análise energéticas, acústicas, luminotécnicas, informações financeiras, estruturais, dentre outros. Neste trabalho, a abordagem do BIM é essencialmente focada na atividade do PAA, considerando essencialmente as etapas de síntese e avaliação do projeto, seguindo os critérios estabelecidos por regulamentos usados pelos órgãos competentes para a avaliação da PAA. Esses regulamentos são textos 
normativos que definem leis, códigos, especificações, normas e assim por diante. Eles geralmente fornecem restrições para soluções ou atividades técnicas que ocorrem sob condições definidas no projeto.

O uso de verificação automatizada ou semiautomatizada pode contribuir significativamente com as atividades de avaliação de projeto, segundo as prescrições estabelecidas nos regulamentos. Assim, torna-se a atividade de avaliação algo mais eficiente, rápido e seguro. O principal objetivo do uso de método como o proposto no artefato apresentado nesse trabalho é o de oferecer modelos computáveis, com sintaxe e semânticas claras que possam ser usadas na criação de requisitos para a avaliação de projetos, no que diz respeito ao cumprimento das regulamentações exigidas pelos órgãos competentes durante a avaliação de PAA.

Guedes e Andrade (2019), a partir da análise do Manual de Critérios e Condicionantes de Planejamento Aeroportuário (INFRAERO,2006), conseguiram identificar uma série de categorias de componentes possíveis e seus respectivos parâmetros que podem ser traduzidos em verificação de regras do código regulatório do Terminal de Passageiros de projetos arquitetônicos. Essas foram agrupadas em 6 categorias e possuem 119 componentes (somente para o Terminal de Passageiros - TPS) que podem ser implementados em um processo automático de verificação de regras BIM.
O fluxo básico do processo de projeto com o BIM pressupõe a integração de vários modelos de informação (representando as diferentes disciplinas de projeto) em um único arquivo. Com esses vários processos de projeto integrados e unificados, o esforço de coordenação é otimizado, facilitando a verificação automática de regras.

A figura 02 apresentada a seguir demonstra um mapa de processo geral de um projeto aeroportuário em BIM. A etapa 01 refere-se ao diagnóstico / documentação a ser desenvolvido pela equipe de analistas referente a listagem das regras, classificação das regras e interpretação das regras e sua estrutura lógica. Já na etapa 02 , uma vez desenvolvidos pela equipe de projetistas os modelos de autoria BIM das várias disciplinas (arquitetura, infraestrutura, estruturas, instalações, ventilação mecânica, etc.) são exportados via IFC formando um Modelo Federado. Na etapa 02, as propriedades contidas no Modelo Federado são validadas conforme o MDM, nesta etapa será realizada uma verificação automática através de um template de regras 01 criado no SMC (ver figura 2) destinado a validar a integridade do modelo IFC, essa avaliação é realizada pela equipe de analistas do projeto aeroportuário. Uma vez validadas as informações referentes a integridade do modelo IFC contidas no modelo integrado, segue para a etapa seguinte (etapa 03). Caso exista alguma incompatibilidade ou ausência de informações, o modelo integrado deve ser revisado novamente pela equipe de projetistas. Uma maneira de aprimorar esse processo de validação, proposto nesse

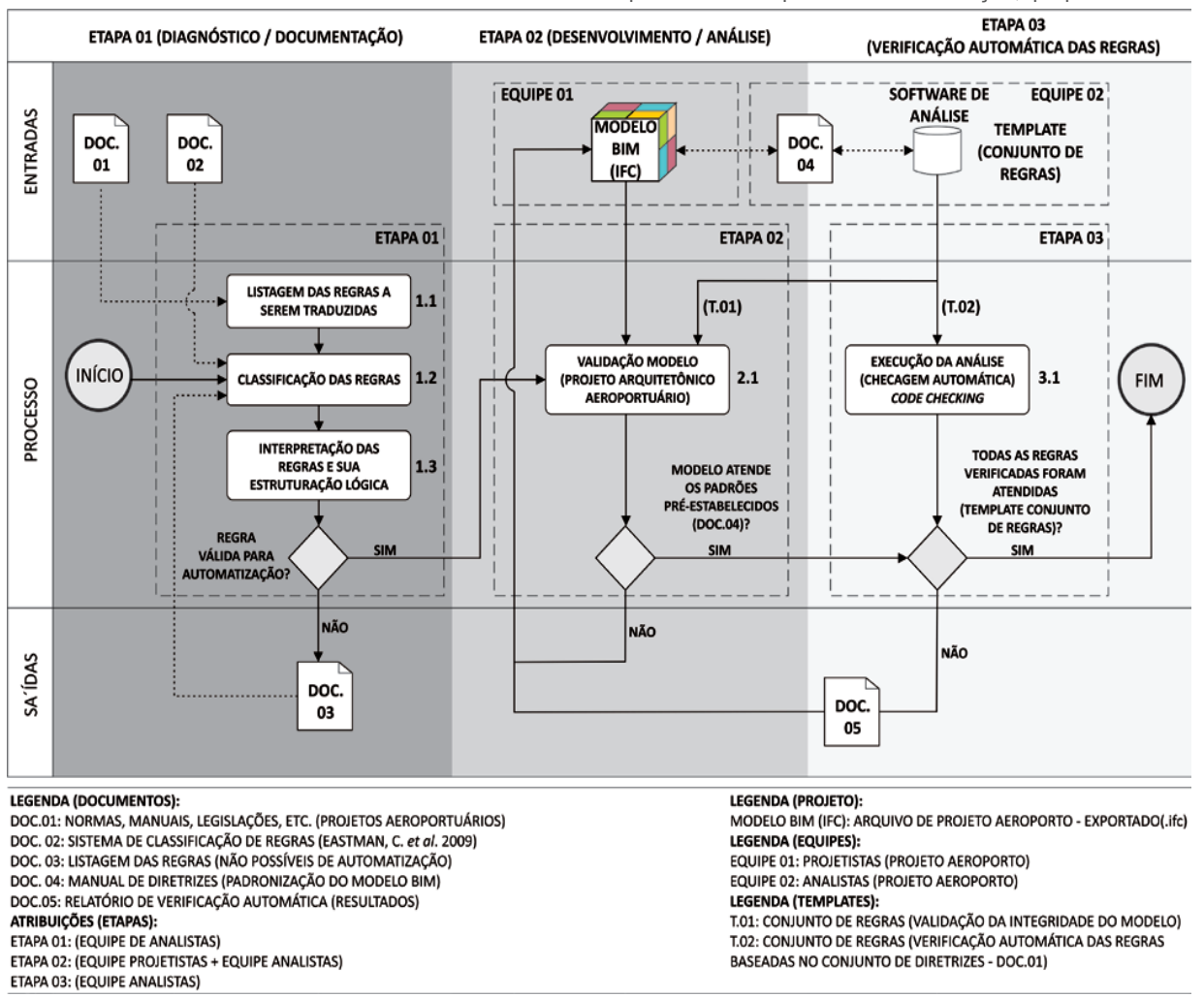

Figura 2: Mapa de processo de avaliação de projeto proposto pela pesquisa. Fonte: Elaborado pelos autores (2020). 
trabalho, é disponibilizando para as equipes de projetistas o MDM (doc.04 apresentado na figura 2) visando subsidiar à padronização das informações contidas do modelo BIM que deverá ser incorporado aos projetos, desde as fases iniciais da elaboração. A intensão do MDM é auxiliar às equipes de projetistas na confecção dos modelos BIM, com diretrizes de modelagem e check-list. A proposta é pendurar a informação correta na posição correta visando a avaliação posterior do projeto pela equipe de analistas de PAA. A Etapa 03 compreende a etapa de verificação de códigos (code checking) no software de análise SOLIBRI Model Checker (SMC). Neste software é realizada a checagem automática com base em um template de regras 02 específico para checagem das regras a serem analisadas no modelo (desenvolvido nessa pesquisa e fornecido à equipe de analistas de projetos aeroportuários). Após a avaliação do modelo é gerado automaticamente um relatório com os resultados obtidos (doc. 05 apresentado na figura 2). Se todas as regras verificadas forem atendidas o processo é concluído, caso contrário, o Relatório de Acompanhamento Técnico (RAT) é enviado para a equipe de projetistas de aeroportos. Esse relatório poderá ser enviado no formato PDF ou por meio de uma plataforma de comunicação BIM (BIMcollab, BIMTrack, por exemplo), baseado no uso do BIM Collaboration Format (BCF). Com base no RAT a equipe reavalia o projeto, altera e envia novamente para avaliação. Tanto a etapa 02 quanto a etapa 03 serão coordenadas pela equipe de analistas de projetos aeroportuários dos órgãos competentes.

Esse artigo traz algumas investigações iniciais sobre como - MDM deve ser criado e organizado. Este propõe diretrizes para padronização de propriedades e dados a serem pendurados no modelo BIM de projetos aeroportuários, possibilitando que as equipes de projetistas possam conceber os seus respectivos projetos em softwares de autoria BIM e que, ao serem enviados para avaliação, esses modelos de informação do projeto possam trazer as informações necessárias e suficientes para uma avaliação precisa, seguindo os critérios estabelecidos pelos documentos normativos de referencia para desenvolvimento de um PAA posibilitando a checagem de regras de maneira eficiente na etapa 03 (ver figura 2).

\section{IFC}

Para possibilitar o intercâmbio de informações entre diferentes modelos BIM, de acordo com Sacks et al. (2018), existem três tipos de formatos para trocas de dados entre aplicativos BIM: 1) link direto; 2) formato de troca proprietário; 3) formatos de arquivo de troca de domínio públicos. O terceiro tipo compreende um padrão aberto de arquivo de troca de informação BIM. Possui propriedades de objetos, medidas, parâmetros, materiais, além de propriedades geométricas. São adequados para uso em aplicativos de análise e gerenciamento de construção. Este artigo investiga a aplicabilidade e padronização do terceiro tipo de troca de dados por meio do esquema IFC em projetos de aeroportos. De acordo com a buildingSMART, O IFC é uma descrição digital padronizada do ambiente construído. É um padrão internacional aberto (ISO 16739-1: 2018), projetado para ser neutro ou independente do fornecedor e usado em uma ampla gama de dispositivos de hardware, plataformas de software e interfaces para muitos casos de uso diferentes. Eastman et al. (2008) definem o esquema da IFC como um modelo de dados padronizado que codifica logicamente a identidade e a semântica (nome, identificador, tipo de objeto etc.); as características ou atributos (material, cor, propriedades etc.); relacionamentos (locais, conexões etc.); objetos (colunas, lajes, vigas, etc.); conceitos abstratos (desempenho, custo, etc.); processos (instalações); operações e pessoas (empresas, designers, contratados, fornecedores, etc.).

O esquema IFC é composto por várias entidades organizadas, como mostrado anteriormente, e com base em três pontos estruturais, IfcObjectDefinition, que define as entidades (objetos), IfcRelationship, que define os relacionamentos entre as entidades e o IfcPropertyDefinition, que define as propriedades associadas às entidades. Segundo Nawari (2018), o IFC é um banco de dados de alto nível que se destina a ser independente de qualquer implementação de software e que deve ser estritamente neutro. Este oferece uma estrutura de dados consistente para armazenar informações de construção, mas não impõe, nem mesmo permite, qualquer método específico de implementá-lo em um aplicativo de software. Atualmente, o esquema IFC (Industry Foundation Classes) é usado para trocar informações de uma parte para outra em uma transação comercial específica. Devido à riqueza de informações que podem ser encapsuladas em atributos, propriedades e geometrias ao longo do ciclo de vida de um edifício e seu ambiente, a quantidade de dados do IFC é muito grande. Para evitar a dificuldade de transferir informações em um modelo IFC, é importante definir meios uniformes e padrões de especificação de conteúdo a serem usados nos estágios do ciclo de vida do edifício. Assim, é importante estabelecer que informação deve ser entregue, por quem, quando e para qual destinatário (Boormann, et al. 2018).

Para resolver isso, a buildingSMART desenvolveu as estruturas do Information Delivery Manual / Model View Definition (buildingSMART, 2013). A ideia é reduzir o espaço para interpretação e facilitar a implementação de estudos de casos em áreas de aplicativos específicas. O Information Delivery Manual (IDM) é um método usado para descrever de forma consistente os processos BIM (IDM, ISO 29481). A estrutura predefinida do IDM e os métodos uniformes para apresentação de modelos de processos permitem que os usuários desenvolvam, concordem e documentem com precisão seus processos BIM. A partir dos dados definidos no IDM, um Model View Definition (MVD) define os subelementos específicos do modelo geral de dados da IFC que podem suportar os requisitos específicos de troca de dados (Steinmann, 2018). Ou seja, um MVD é uma recomendação para quais dados e elementos o modelo IFC deve incluir, dependendo da finalidade da troca do modelo. Como exemplo de MVD, podemos citar: Coordination View 2.0, FM Handorver View, Structural Analysis View, entre outros.

Em geral, as trocas de dados usadas nos sistemas de verificação de regras devem atender a certos requisitos. Para este trabalho, são considerados requisitos essenciais: 1) Uso de ferramentas BIM apropriadas para modelagem de objetos. Por exemplo, a criação de uma "parede" deve ser feita usando a ferramenta "parede" do software de modelagem; 2) garantir que todas as 
nomenclaturas correspondam às estabelecidas nos manuais. Por exemplo, para os nomes dos ambientes, use os nomes estabelecidos nos manuais da INFRAERO (sala de embarque, fila de check-in, etc.) e 3) Verificar se o mapeamento do IFC e suas ferramentas nativas de criação de BIM estão corretamente configuradas para exportar o modelo IFC que esteja em conformidade com o MVD estabelecido pelos sistemas de verificação de regras a serem usados em projetos aeroportuários. Nesse caso, o Coordination View 2.0 será o MVD inicialmente adotado.

\section{RESULTADOS}

Os estudos iniciais desta pesquisa, apresentados neste artigo, apontam para a importância de padronizar os Modelos BIM desenvolvidos pelos diversos projetistas visando a avaliação do projeto pela equipe de analistas de PAA. Para chegar a esses resultados iniciais, foram realizadas simulações no projeto de um terminal de passageiros aeroportuário (TPS), modelado em um software de autoria BIM. Essas simulações mostram, mesmo de forma preliminar, a viabilidade de padronizar determinados parâmetros de projeto de aeroportos para verificar a conformidade com os requisitos (Etapa 03 Figura 02). Esses estudos também indicam a necessidade de criar um MDM, já citado anteriormente. Espera-se que a partir do uso dos protocolos definidos no MDM pela equipe de projetistas as informações BIM estejam corretamente penduradas no modelo, de modo a que sejam validadas na Etapa 02 (Figura 02).

O ponto de partida da pesquisa foi analisar o processo de avaliação desenvolvido por equipes de analistas de projeto de aeroportos no Brasil. Os resultados indicam que foi possível identificar alguns pontos importantes em relação ao processo de avaliação do projeto atualmente em vigor (analógico): 1) a qualidade da análise está diretamente associada à maturidade do analista; 2) existência de ambiguidade no processo de análise de projetos de aeroportos tendo em vista a falta de padronização da análise, baseada na percepção de cada analista; 3) tempo de análise estendido, considerando que a maioria dos projetos de aeroportos brasileiros ainda é submetida para análises em desenhos CAD bidimensionais (desenhos assistidos por computador).

Por outro lado, os primeiros estudos dessa pesquisa mostraram que, através da verificação de códigos identificar a possibilidade de melhorar o processo atual de avaliação de projetos de aeroportos. Porém, para isso, além do desenvolvimento do template de regras no SMC foi necessário desenvolver um documento para padronizar diretrizes para modelagem de projetos no software BIM, o MDM. Através da aplicação das regras estabelecidas nos manuais, que podem ser incorporadas nos processos de modelagem por parte das equipes de projeto e de uma exportação usando um MVD apropriado, os Modelos BIM produzidos pelos projetistas podem estar aptos a serem checados por meio de um software de checagem automática de regras. Esta é a última etapa executada pelos analistas dos órgãos competentes responsáveis pelas avaliações dos projetos (Etapa 03 - Figura 01).

O estágio atual da pesquisa consiste em verificar as regras de avaliação automática e identificar as propriedades mínimas do IFC a serem penduradas no modelo BIM. Duas situações de avaliação são apresentadas neste artigo. Estas foram simuladas com base nos componentes existentes no Manual de Critérios e Condicionantes de Planejamento Aeroportuário (INFRAERO, 2006) e da NBR 9050 (2015). Cada regra simulada foi classificada de acordo com as classes gerais de regras recomendadas por Solihin e Eastman (2015), sendo: classe 01 - regras que requerem um número único ou pequeno de dados explícitos; classe 02 - regras que requerem valores simples de atributos derivados; classe 03 - regras que requerem estrutura de dados estendida; e classe 04 - regras que exigem uma "prova de solução". A seguir, serão mostrados exemplos, já implementados (consulte a Figura 03 e Figura 04), que apresentam a descrição da verificação de regras, a classificação das regras e as propriedades mínimas necessárias no modelo BIM IFC do projeto.

A Figura 03 descreve a simulação da regra 01 apresentada neste trabalho. A condição de aceitação desta regra é: a área livre mínima em um sanitário acessível deve ser $\geq$ $1,40 \mathrm{~m}$. Se o modelo IFC for compatível, o software de análise (SMC) mostrará o resultado gráfico representando um círculo verde no piso (compatível), caso contrário, o resultado gráfico no componente verificado não será compatível, o SMC mostrará o resultado gráfico representando um círculo vermelho no piso (não compatível). Para a regra 01 , a condição não era adequada e, portanto, o SMC identificou a não conformidade com a regra estabelecida. Neste caso, é necessário que o projeto aeroportuário seja corrigido pela

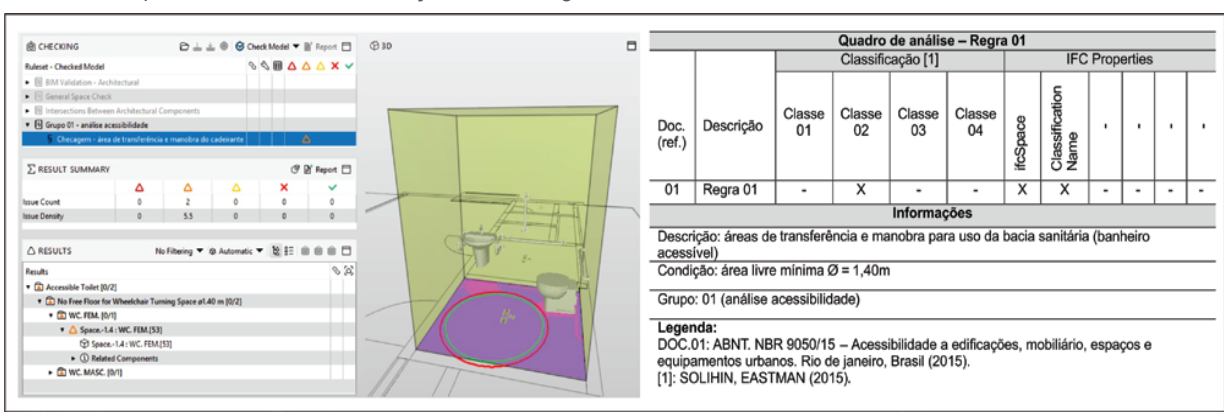

Figura 3: Checagem automática da regra 01 no SMC (lado esquerdo) - Quadro de análise da regra 01 (lado direito). Fonte: Elaborado pelos autores (2020).

regulatórios e da padronização do modelo de informação equipe de projetistas e reenviado para nova avaliação. no software BIM (usando o formato IFC), é possível 
A figura 04 apresentada na página seguinte, refere-se à simulação da regra 02 apresentada neste trabalho. A condição de aceitação desta regra é que a profundidade mínima do balcão de atendimento do check-in seja $\geq 4,00$ $\mathrm{m}$. Se o modelo IFC for compatível, o software de análise (SMC) mostrará o resultado gráfico em verde (compatível), propriedades IFC sejam padronizadas no estágio de criação dos modelos, de acordo com os parâmetros estabelecidos nas regras de verificação do modelo (quadro de análise). Além disso, este deve ser exportado no IFC usando os protocolos definidos nos manuais (por exemplo, usando o MVD apropriado). Na regra 02 apresentada na

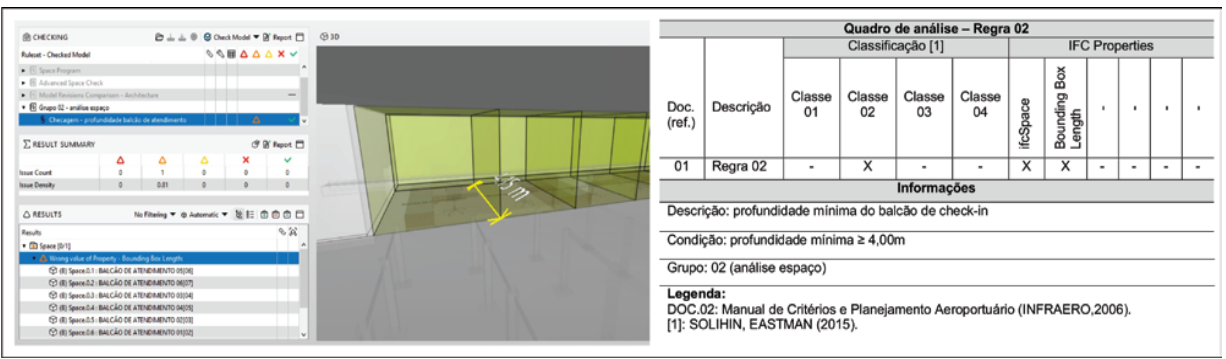

Figura 4: Checagem automática da regra 02 no SMC (lado esquerdo) - Quadro de análise da regra 02 (lado direito). Fonte: Elaborado pelos autores (2020).

caso contrário, o componente verificado não será compatível e o SMC mostrará o resultado gráfico em vermelho (não compatível). Para a regra 02, a condição estava em conformidade; portanto, o SMC identificou a conformidade com a regra estabelecida, sem a necessidade de alterar o projeto.

O que pode ser visto com esses dois exemplos é que, assim como é importante estabelecer regras para a avaliação de modelos de informação de edifícios, a criação de regras por si só é insuficiente para que o processo de avaliação seja eficiente. Para que as regras sejam efetivamente lidas e validadas ou não (verdadeiras ou
Figura 04, por exemplo, entidades como IfcSpace, Boundign Box Length foram consideradas. Para que o SMC pudesse avaliar a regra transcrita na figura 04, o modelo de informação precisou conter essas entidades. Qualquer outra informação diferente do padrão estabelecido no quadro de análise resultaria na impossibilidade de avaliar esta regra no SMC. Esses dois exemplos apresentados neste documento demonstram, mesmo de maneira preliminar, a importância de padronizar as entidades do IFC nos Modelos BIM durante o processo de projeto. Ou seja, para que a equipe de avaliação possa executar com êxito o processo automatizado de verificação de regras usando o software de análise BIM, o

\begin{tabular}{l}
\hline \multicolumn{2}{|c|}{ Quadro Sintese - Checagem de Regras } \\
\hline
\end{tabular}

Figura 4: Quadro Síntese - Checagem das Regras. Fonte: Elaborado pelos autores (2020).

falsas), os Modelos BIM dos projetos devem ser organizados de modo que as informações presentes nas modelo BIM deve conter as entidades IFC padronizadas, incorporadas ao modelo BIM. Essas entidades devem ser 
incorporadas corretamente no processo de desenvolvimento do projeto, de acordo com os parâmetros criados no modelo de regras implementado no software de avaliação. Para isso, é necessário conhecer a estrutura organizacional das informações presentes no software de avaliação, para que seja possível sobrepor os dados do IFC ao conjunto de regras criadas. Faz-se necessário que os modelos BIM de um PAA, desenvolvidos pelos projetistas incorporem as entidades IFC pré-estabelecidas pela equipe de analistas para que o software de análise possa executar corretamente a checagem de regras de maneira automatizada.

A figura 05 apresentada na página anterior demonstra um quadro síntese com as informações referentes às duas regras apresentadas neste artigo. Uma vez mapeadas todas as informações necessárias pela equipe de analistas de um PAA, é possível através de um quadro síntese categorizar cada regra mediante seu grupo específico, classificar as regras de acordo com as classes de regras definidas pro Solihin e Eastman (2015), correlacionar os parâmetros IFC necessários ou não para cada regra específica, assim como, identificar os parâmetros específicos para criação das regras no software de avaliação BIM. Assim, esse quadro síntese servirá de base para a confecção do Manual de Diretrizes para a Modelagem em BIM. Esse manual incorporará informações de legislação, suas transcrições em regras, as classes que cada uma das regras se encontram e as propriedades IFC vinculadas a cada uma das regras. Além do mais, esse manual apresentará também os protocolos para exportação dos Modelos BIM por parte das empresas de projeto e o tecerá informações sobre o MDV a ser utilizado no momento de exportar o arquivo IFC. Desse modo, a partir da consulta do MDM a equipe de projetistas de PAA poderá incorporar nos seus templates e nas suas práticas de projeto e modelagem as prescrições definidas no MDM. Dessa forma, espera-se que esses modelos possam estar adequados para a verificação automática, tanto na Etapa 02, quanto na Etapa 03 (Figura 02). A verificação em duas etapas, sendo a primeira de avaliação da integridade do modelo IFC e a segunda de atendimento do modelo aos parâmetros normativos que norteiam o desenvolvimento de projetos aeroportuários no Brasil, permitirá uma maior qualidade no processo de avaliação, evitando retrabalhos e garantindo maior assertividade nos processos de avaliação automática usando CC. É importante notar que os exemplos das regras apresentadas nesta pesquisa se referem às regras da classe 02 com base em Solihin e Eastman (2015), ou seja, são regras que requerem valores de atributos derivados simples. Isso não significa que esse método não possa beneficiar analistas na validação das classes de regras 03 e 04. Um exemplo clássico do uso dessas últimas regras é o chamado Design Assessment Tool (DAT). Essa ferramenta, desenvolvida pelo Instituto de Tecnologia da Geórgia, usou as regras de circulação e segurança encontradas no US Courts Design Guide (CDG, 2007) que as codifica em 302 regras paramétricas computáveis (Eastman, 2009). Um exemplo de regra da classe 04, para o caso de TPS, seria o analista avaliar como um funcionário do aeroporto faria um caminho de acesso através da área de serviço específica de um determinado sistema dentro do TPS com seu equipamento para realizar manutenção ou reparo em um sistema específico.
A definição das classes de regras é também um ponto importante tratado por essa pesquisa. As regras classes 3 e 4 são regras mais complexas e que exigem um esforço maior na etapa de modelagem e na avaliação. Para essas regras, estão sendo analisados os níveis de esforços desprendidos para automatização da avaliação. Espera-se com isso identificar em todas as categorias de regras estudadas aquelas que serão automatizadas, aqui se enquadram principalmente as regras de classe 01 e 02 , aquelas que serão semiautomatizadas e aquelas que permanecerão com uma avaliação manual. A partir do momento em que é possível automatizar ou semiautomatizar o processo de avaliação do projeto de PAA em um conjunto de regras, sejam elas simples ou mais complexas, acredita-se que os analistas terão mais tempo para lidarem com questões mais subjetivas de projeto, tais como, análise de funcionalidades relacionadas a atividades operacionais, manutenção predial e processos operacionais, dentre outros aspectos relevantes e subjetivos. No processo de avaliação atual (manual), os analistas passam considerável tempo na fase de avaliação, verificando uma série de componentes com potencial para serem implementados em um processo de Verificação de Código. Um exemplo de possíveis componentes a serem implementados em um processo de verificação automática são os itens do projeto arquitetônico de um terminal de passageiros aeroportuário, verificados por Guedes e Andrade (2019).

\section{CONCLUSÃO}

Esta pesquisa, ainda em fase de desenvolvimento, demonstra a importância de criar um processo de padronização na modelagem de informações durante o desenvolvimento de projetos de aeroportos em software de autoria do BIM, considerando a exportação para um modelo IFC integrado. Tendo em vista a inexistência de manuais sobre esse assunto no Brasil, é possível observar o potencial dessa abordagem e, em breve, beneficiar todas as equipes de projetistas e analistas de aeroportos. Após a criação do Manual de Diretrizes de Modelagem (padronização do modelo BIM), com base nos padrões técnicos para esse tipo de projeto, este manual beneficiará os profissionais envolvidos no processo de síntese e avaliação dos projetos em vários pontos. As equipes que trabalham no desenvolvimento do projeto (síntese) terão um documento de referência, durante o processo de projeto, para padronizar quais tipos de informações o modelo BIM deverá carregar e como essas informações deverão estar penduradas em um arquivo IFC, minimizando a probabilidade do envio de modelos BIM com informações incompletas ou inconsistentes. Da mesma forma, os analistas poderão, por meio da verificação de regras, automatizarem grande parte do processo atualmente predominantemente manual, aproveitando a economia de tempo, aumentando a eficiência no processo de avaliação, minimizando a ambiguidade, padronizando o processo de avaliação, dentre outros benefícios relacionados à fase de aprovação do projeto. Além disso, é claro, a possibilidade de uso posterior do modelo IFC para manutenção e operação do aeroporto. Atualmente a pesquisa está mapeando as regras mais importantes, ou seja, aquelas mais recorrentes nos processos de avaliação e que exigem um menor esforço em implementar. Esse mapeamento está sendo realizado através de entrevistas e coleta de documentos 
junto às empresas responsáveis pelo desenvolvimento e aprovação de projetos. Definido e mapeado o artefato, a etapa seguinte consistirá na validação do artefato proposto, junto à essas mesmas empresas.

Durante as entrevistas e desenvolvimento do projeto foi possível observar o potencial em usar os parâmetros do software de análise para outros tipos de avaliação de projetos de aeroportos, além dos abordados nesta pesquisa. Existem elementos de avaliação de projeto, como análise de zoneamento de ruído, análise de zona de proteção, análise de rampa de transição lateral, rampa de aproximação, dentre outros itens, que também podem ser personalizados em um conjunto de regras específicas para analisar esses parâmetros que em muitos casos, ultrapassam os limites do sítio aeroportuário.

\section{REFERÊNCIAS}

ABNT. NBR 9050/15 (2015). Acessibilidade a edificações, mobiliário, espaços e equipamentos urbanos. Rio de janeiro, Brasil.

BIM ThinkSpace (2015). Episode 24: Understanding Model Uses. v0.73.

Borrmann, A.; Köning, M.; Koch, C.; Beetz, J. (Eds.) Building Information Modeling: Technology Foundations and Industry Practice. 1st. edn. Springer, Cham, pp. 81-126 (2018).

BuildingSMART (2013). Construction operations building information exchange, MVD definition for IFC4.
Eastman, C., Lee, J., Jeong, Y., Lee, J. (2009). Automatic rulebased checking of building designs. Automation in Construction, v.18, n.8, p.1011-1033.

Guedes, İtalo; Andrade, Max. (2019). Automatic Rule-Based Checking for the Approval of Building Architectural Designs of Airport Passenger Terminals based on BIM. Proceedings of the 37th eCAADe and 23rd SIGraDi Conference - Volume 2, University of Porto, Porto, Portugal, 11-13, 333-338.

ISO - International Organization For Standardization (2018). ISO 16739-1: 2018 - Industry Foundation Classes (IFC) for data sharing in the construction and facility management industries.

Nawari, O.N. (2018). Building information modeling: automated code checking and compliance processes (1th ed.). CRC Press, Boca Raton.

Sacks, R., Eastman, C., Lee, G., Teicholz, P. (2018). BIM Handbook: a Guide to Building Information Modeling for Owners, Managers, Designers, Engineers, and Contractors. (3th. ed). New Jersey: John Wiley \& Sons.

Solihim, W: Eastman, C. (2015). Classification of rules fo automated BIM rule checking development. Automation in construction, 53, pp.69-82

Steinmann, R. (2018). IFC Certification of BIM Software. In: Borrmann, A.; Köning, M.; Koch, C.; Beetz, J. (Eds.) Building Information Modeling: Technology Foundations and Industry Practice. 1st. edn. Springer, Cham, pp. 139-154

Superintendência de Empreendimentos de Engenharia - DEEP Gerência de Planejamento de Engenharia - EPPL (2006). Manual de Critérios e Condicionantes de Planejamento Aeroportuário. 